\title{
Assessing Snow Albedo Feedback in Simulated Climate Change
}

\author{
Xin Qu And Alex Hall \\ Department of Atmospheric and Oceanic Sciences, University of California, Los Angeles, Los Angeles, California
}

(Manuscript received 23 January 2005, in final form 17 August 2005)

\begin{abstract}
In this paper, the two factors controlling Northern Hemisphere springtime snow albedo feedback in transient climate change are isolated and quantified based on scenario runs of 17 climate models used in the Intergovernmental Panel on Climate Change Fourth Assessment Report. The first factor is the dependence of planetary albedo on surface albedo, representing the atmosphere's attenuation effect on surface albedo anomalies. It is potentially a major source of divergence in simulations of snow albedo feedback because of large differences in simulated cloud fields in Northern Hemisphere land areas. To calculate the dependence, an analytical model governing planetary albedo was developed. Detailed validations of the analytical model for two of the simulations are shown, version 3 of the Community Climate System Model (CCSM3) and the Geophysical Fluid Dynamics Laboratory global coupled Climate Model 2.0 (CM2.0), demonstrating that it facilitates a highly accurate calculation of the dependence of planetary albedo on surface albedo given readily available simulation output. In all simulations it is found that surface albedo anomalies are attenuated by approximately half in Northern Hemisphere land areas as they are transformed into planetary albedo anomalies. The intermodel standard deviation in the dependence of planetary albedo on surface albedo is surprisingly small, less than $10 \%$ of the mean. Moreover, when an observational estimate of this factor is calculated by applying the same method to the satellite-based International Satellite Cloud Climatology Project (ISCCP) data, it is found that most simulations agree with ISCCP values to within about $10 \%$, despite further disagreements between observed and simulated cloud fields. This suggests that even large relative errors in simulated cloud fields do not result in significant error in this factor, enhancing confidence in climate models. The second factor, related exclusively to surface processes, is the change in surface albedo associated with an anthropogenically induced temperature change in Northern Hemisphere land areas. It exhibits much more intermodel variability. The standard deviation is about $1 / 3$ of the mean, with the largest value being approximately 3 times larger than the smallest. Therefore this factor is unquestionably the main source of the large divergence in simulations of snow albedo feedback. To reduce the divergence, attention should be focused on differing parameterizations of snow processes, rather than intermodel variations in the attenuation effect of the atmosphere on surface albedo anomalies.
\end{abstract}

\section{Introduction}

As surface air temperature increases in climate change simulations with coupled land-ocean-atmosphere models, snow over the Northern Hemisphere $(\mathrm{NH})$ extratropics retreats, revealing land surface that is much less reflective of solar radiation. The additional absorbed solar radiation results in more warming $(\mathrm{Cu}-$ basch et al. 2001; Holland and Bitz 2003). This positive feedback, the so-called snow albedo feedback, accounts for about half of the additional net incoming solar radiation associated with the $\mathrm{NH}$ cryosphere retreat in

Corresponding author address: Xin Qu, Dept. of Atmospheric and Oceanic Sciences, University of California, Los Angeles, Los Angeles, CA 90095.

E-mail: xinqu@atmos.ucla.edu equilibrium climate change simulations (Hall 2004). The satellite record also supports the idea of a positive snow albedo feedback. By correlating Earth Radiation Budget Experiment top-of-the-atmosphere shortwave fluxes and satellite-observed snow extent in the $\mathrm{NH}$ extratropics, Groisman et al. (1994a,b) pointed out that a large portion of the $\mathrm{NH}$ warming for the last two decades of the twentieth century can be attributed to snow albedo changes. Despite the consensus on the sign of the feedback, its strength as seen in climate change simulations carried out over the past two decades varies significantly (Cess et al. 1991; Randall et al. 1994; Cubasch et al. 2001; Holland and Bitz 2003). These differences become a source of divergence in simulated climate change (Cubasch et al. 2001; Holland and Bitz 2003).

According to the classic climate sensitivity frame- 
work (e.g., Cess and Potter 1988), the strength of the snow albedo feedback in climate change can be quantified by the variation in net incoming shortwave radiation $Q$ with surface air temperature $T_{s}$ because of changes in surface albedo $\alpha_{s}$ :

$$
\left(\frac{\partial Q}{\partial T_{s}}\right)_{\mathrm{SAF}}=\frac{\partial Q}{\partial \alpha_{s}} \frac{\Delta \alpha_{s}}{\Delta T_{s}},
$$

where the subscript SAF is used to emphasize that the partial derivative refers only to changes in solar radiation with surface temperature that occur because of changes in the snowpack, rather than changes in cloudiness or other factors that could affect solar radiation. According to Eq. (1), snow albedo feedback is the product of two terms, one representing the dependence of net incoming solar radiation on surface albedo $\left(\partial Q / \partial \alpha_{s}\right)$, and another representing the change in surface albedo induced by a unit temperature change $\left(\Delta \alpha_{s} / \Delta T_{s}\right)$.

The partial derivative, $\partial Q / \partial \alpha_{s}$ [first term in Eq. (1)] can be rewritten as follows:

$$
\frac{\partial Q}{\partial \alpha_{s}}=-I_{t} \frac{\partial \alpha_{p}}{\partial \alpha_{s}},
$$

where $I_{t}$ is the incoming solar radiation at the top of atmosphere (TOA), which we take to be a constant, and $\partial \alpha_{p} / \partial \alpha_{s}$ is the variation in planetary albedo with snow albedo, with other factors such as water vapor concentration and cloud fixed. It represents the attenuation effect of the atmosphere on snow albedo anomalies, which results from two processes. First, incoming solar photons at the TOA are partly absorbed and reflected back to space by the atmosphere, reducing the number reaching the surface. Second, solar photons initially reflected by the surface are partly absorbed and reflected back to the surface by the atmosphere, reducing the number reaching the TOA. Obviously the more the atmosphere absorbs and scatters solar radiation because of factors such as water vapor concentration and cloudiness, the greater the atmospheric attenuation of snow albedo anomalies. Because mean water vapor concentration and cloudiness differ among climate models, values of $\partial \alpha_{p} / \partial \alpha_{s}$ may vary from model to model, creating divergence in the strength of simulated snow albedo feedback quite apart from divergence that arises from differing simulations of surface processes, represented by the second term in Eq. (1), $\Delta \alpha_{s} / \Delta T_{s}$. We wish to isolate and assess this divergence in $\partial \alpha_{p} / \partial \alpha_{s}$ values. Cloud changes associated with cloud feedback processes also result in changes in the attenuation effect of the atmosphere on snow albedo anomalies, and in turn may cause $\partial \alpha_{p} / \partial \alpha_{s}$ to vary significantly with time as the overall climate changes. This may make it difficult to use a single value to represent the strength of this aspect of snow albedo feedback. We wish to determine whether this temporal variation is important.

We also aim to isolate and assess the divergence resulting from the second term of Eq. (1), $\Delta \alpha_{s} / \Delta T_{s}$. However, this term is straightforward to quantify from model output by simply dividing the simulated change in $\alpha_{s}$ by the change in $T_{s}$. The first term, on the other hand, is difficult to calculate directly from model output because it is a partial derivative assuming fixed clouds and water vapor. Previous efforts have focused on the ratio, $\Delta \alpha_{p} / \Delta \alpha_{s}$, rather than $\partial \alpha_{p} / \partial \alpha_{s}$ (Cess et al. 1991; Randall et al. 1994), where $\Delta \alpha_{s}$ and $\Delta \alpha_{p}$ represent simulated changes in surface and planetary albedo from the current climate to the future warmer climate, calculated from TOA and surface fluxes commonly stored by climate simulations. However, this approach conflates model divergence stemming from cloud feedback with that stemming from snow albedo feedback, since cloud changes contaminate TOA solar fluxes used to calculate planetary albedo.

Because of this difficulty, the focus of much of this paper is isolating the $\partial \alpha_{p} / \partial \alpha_{s}$ component of the snow albedo feedback, and is done by developing an analytical model of planetary albedo. In the analytical model, planetary albedo is represented as a function of known model variables, such as surface albedo, cloud cover, and cloud optical thickness. We determine the coefficients of the terms relating these variables to planetary albedo empirically from model output. With the analytical model, calculation of a true partial derivative with respect to surface albedo is straightforward. Our results rest upon the ability of the analytical model to capture simulated variations in planetary albedo. We therefore validate it in transient climate change simulations with several new versions of state-of-the-art models of the Fourth Assessment Report (AR4) of the Intergovernmental Panel on Climate Change (IPCC) and find that it does remarkably well. This allows us to use the analytical model to calculate values of $\partial \alpha_{p} / \partial \alpha_{s}$ in these models with a high degree of accuracy. Moreover, we find we can apply the analytical model with the same high degree of accuracy to the International Satellite Cloud Climatology Project (ISCCP) data from 1984 to 2000. This facilitates the use of satellite data to assess the realism of the simulations. Though we validate the analytical model in all the simulations, for simplicity we show details only for two of the models, version 3 of the National Center for Atmospheric Research (NCAR) Community Climate System Model (CCSM3), and the Geophysical Fluid Dynamics Laboratory (GFDL) global coupled Climate Model 2.0 (CM2.0). Our study focuses on springtime [March-May (MAM)]. The reason for this is that over half the in- 
TABLE 1. A list of 17 models used in this study and the numbers they correspond to in Figs. 6 and 7. For the documentation of the models, see http://www-pcmdi.llnl.gov/ipcc/model_ documentation/ipcc_model_documentation.php.

\begin{tabular}{cl}
\hline \hline Number & \multicolumn{1}{c}{ Model acronym } \\
\hline 1 & CNRM-CM3, France \\
2 & MRI CGCM2.3.2, Japan \\
3 & GISS-ER, United States \\
4 & CSIRO Mk3.0, United States \\
5 & PCM, United States \\
6 & UKMO-HadCM3, United Kingdom \\
7 & CGCM3.1 (T47), Canada \\
8 & FGOALS-g1.0, China \\
9 & ECHAM5-MPI/OM, Germany \\
10 & UKMO-HadGEM1, United Kingdom \\
11 & ECHO-G, Germany/Korea \\
12 & IPSL-CM4, France \\
13 & CCSM3, United States \\
14 & MIROC3.2 (medres), Japan \\
15 & INM-CM3.0, Russia \\
16 & GFDL-CM2.0, United States \\
17 & GFDL-CM2.1, United States
\end{tabular}

crease in net incoming solar radiation associated with snow retreat occurs during this season in climate change simulations (Hall 2004), as both snow extent and incoming solar radiation are relatively large. However, the methods we develop here can be easily extended to other seasons.

Our results are presented as follows: A brief description of climate models and ISCCP datasets is given in section 2. In section 3 , we illustrate how both surface albedo and clouds contribute to the planetary albedo change from the current to the future climate in the $\mathrm{NH}$ extratropics using the GFDL CM2.0 and the NCAR CCSM3 simulations as examples. An analytical model for planetary albedo is developed and validated against simulated and ISCCP datasets in section 4 . In section 5 , we use this model to obtain an expression for $\partial \alpha_{p} / \partial \alpha_{s}$ and assess it in the current and future climate based on simulated and ISCCP datasets. Summary and implications are found in section 6 , where we also present calculations of the $\Delta \alpha_{\mathrm{s}} / \Delta T_{s}$ term of Eq. (1) for the AR4 models. In this section we compare the intermodel divergence in this term with the divergence in the $\partial \alpha_{p} / \partial \alpha_{s}$ term to see which accounts for the most overall intermodel divergence in simulations of snow albedo feedback.

\section{Models and observations}

\section{a. Models}

We use output from the twentieth and twenty-second centuries of transient climate change experiments with
17 AR4 models (see Table 1). The twentieth-century climate is simulated in these models by imposing estimates of climate forcings from the period 1901-2000, while the twenty-second century is simulated by imposing the IPCC's A1B Emission Scenario (Prentice et al. 2001).

Since the analytical model is validated in detail for only the NCAR CCSM3 and GFDL CM2.0 models, only these two models are briefly described here. The NCAR CCSM3 (referred to hereafter as CCSM3) consists of general circulation models of the atmosphere and ocean, a land surface model, and a sea ice model. The atmospheric component (Collins et al. 2004) has a horizontal resolution of approximately $1.4^{\circ}$ latitude by $1.4^{\circ}$ longitude and 26 vertical finite difference levels. The land surface component contains a simplified treatment of surface processes responsible for land-atmosphere exchange of heat, water, and momentum (Oleson et al. 2004). A canopy radiative transfer scheme is used to calculate surface albedo, accounting for the joint albedo effect of canopy and ground. Ground albedo is a weighted combination of soil and snow albedos by snow cover, which in turn is a function of snow depth. Snow albedo is parameterized as a function of solar zenith angle and snow age. The oceanic component (Smith and Gent 2004) has $320 \times 384$ points horizontally and 40 ocean levels vertically. The sea ice component includes a subgrid-scale ice thickness distribution, energy conserving thermodynamics, and elasticviscous-plastic dynamics (Briegleb et al. 2004).

Similar to CCSM3, the GFDL CM2.0 (referred to hereafter as CM2.0) consists of general circulation models of the atmosphere and ocean, a land surface model, and a sea ice model. The atmosphere component uses a new grid point dynamical core, a prognostic cloud scheme, and a multispecies aerosol climatology (GAMDT 2004). It has a horizontal resolution of $2.5^{\circ}$ longitude by $2^{\circ}$ latitude and 24 vertical levels. The land component is based on the land dynamics model described by Milly and Shmakin (2002). Surface albedo over snow-covered regions is parameterized as an average of snow-free surface albedo and snow albedo, which are weighted by snow cover. Snow cover is in turn a function of snow depth. Snow albedo is parameterized as a function of surface temperature. This scheme does not explicitly include canopy effects in its calculation of surface albedo, as is the case with CCSM3. The model has a full three-dimensional dynamical ocean and a complex sea ice model; however, no official documentation of the ocean and sea ice components of CM2.0 was available when this paper was composed. 


\section{b. ISCCP datasets}

The ISCCP D-series cloud datasets used in this study are based on observations from a suite of operational weather satellites measuring the temporal and spatial distribution of visible (VIS; wavelength $\approx 0.6 \mu \mathrm{m}$ ), near-infrared (NIR; wavelength $\approx 3.7 \mu \mathrm{m}$ ), and infrared (IR; wavelength $\approx 11 \mu \mathrm{m}$ ) radiation. These measurements are then employed to retrieve information about clouds, such as cloud cover, cloud optical thickness, and cloud-top pressure (Rossow and Schiffer 1991; Rossow and Garder 1993a,b; Rossow et al. 1993; Rossow and Schiffer 1999). Three changes have been made in the D-series datasets to enhance the accuracy of cloud detection over snow- and ice-covered surfaces (Rossow and Schiffer 1999): 1) most importantly, a new threshold test on $3.7-\mu \mathrm{m}$ radiances was used, exploiting significantly greater contrast between cloudy and clear scenes over snow- and ice-covered surfaces at this frequency than at $0.6 \mu \mathrm{m} ; 2$ ) at the high latitudes, the visible radiance threshold test was changed to a visible reflectance threshold test; 3 ) over snow and ice in the polar regions, both the VIS and IR thresholds were lowered. Together these improvements have been shown to significantly increase low-level cloud detection sensitivity over snow and ice and reduce the biases in cloud optical thickness of previous ISCCP C-series datasets in these regions.

Accompanying the ISCCP D-series cloud datasets are radiative flux datasets containing solar and infrared radiative fluxes at the TOA and surface for both clearsky and full-sky situations. They are calculated by specifying the following information in a radiative transfer model (Zhang et al. 2004): 1) atmospheric temperature and humidity profiles; 2) vertical profiles of various atmospheric gases, such as $\mathrm{CO}_{2}, \mathrm{O}_{3}, \mathrm{O}_{2}$, and $\mathrm{CH}_{4}$; 3) vertical aerosol profiles for the troposphere and stratosphere; 4) ISCCP D-series cloud datasets; and 5) snow and ice cover data. All the data mentioned above are time-varying so that observed variations in radiative properties of the atmosphere and surface are reflected in the fluxes at the TOA and surface.

These datasets are provided on a global $2.5^{\circ} \times 2.5^{\circ}$ grid and cover the period from 1984 to 2000.

\section{Simulated reduction in planetary albedo over NH extratropical land areas}

In this section, we demonstrate how both surface albedo and clouds contribute to the planetary albedo change from the current to the future climate in $\mathrm{NH}$ extratropical land areas using the CM2.0 and CCSM3 simulations as examples.

Figure 1 shows simulated changes in springtime $\alpha_{s}$, cloud cover $(c)$, logarithm of cloud optical thickness $\ln (\tau+1)$, and $\alpha_{p}$ from the twentieth- to the twentysecond-century climate in the $\mathrm{NH}$ extratropics. We show the logarithm of $\tau$, rather than $\tau$ itself, to take account of the quasi-logarithmic dependence of cloud albedo on $\tau$ (Rossow et al. 1996). Because cloud liquid water path $c_{\text {lwp }}$ and ice water path $c_{\text {iwp }}$ rather than $\tau$ are provided by the CM2.0 and CCSM3 datasets, a relation is used to convert $c_{\mathrm{lwp}}$ and $c_{\mathrm{iwp}}$ into $\tau$ :

$$
\tau=\kappa_{1} c_{\mathrm{lwp}}+\kappa_{2} c_{\mathrm{iwp}},
$$

where $\kappa_{1}$ and $\kappa_{2}$ are assumed to be 0.16 and 0.10 , respectively, in accordance with the values used by the ISCCP (Rossow et al. 1996). This facilitates the comparison between ISCCP datasets and model datasets in the current climate in sections 4 and 5 .

As shown in Fig. 1, surface albedo decreases in the warmer climate in both CM2.0 and CCSM3 throughout the NH extratropical land areas, especially northern Canada, western Russia, and the Tibetan Plateau. However, the decrease is much greater in CM2.0 than CCSM3. The surface albedo reductions averaged over extratropical North America and Eurasia in CM2.0 are about $5 \%-8 \%$, but are only about $3 \%-4 \%$ in CCSM3 (see Fig. 2). The surface albedo reduction in CCSM3 is smaller because surface albedo is less sensitive to the change in surface air temperature and also because the overall warming is smaller in the model. As shown in Table 2 , the ratio $\Delta \alpha_{\mathrm{s}} / \Delta T_{s}$ in CCSM3 is about $10 \%$ and $50 \%$ smaller than that of CM2.0 in extratropical Eurasia and North America, respectively.

Changes in cloud cover and cloud optical thickness simulated in CM2.0 and CCSM3 differ not only in amplitude, but also in sign. Cloud cover in CM2.0 decreases in the warmer climate nearly everywhere in North America and Eurasia, especially in the western United States and western Europe. This reduction, averaged over extratropical North America and Eurasia, is about $1 \%$ and $4 \%$, respectively (see Fig. 2 ). In contrast, cloud cover simulated in CCSM3 increases in the high latitudes of Eurasia and nearly everywhere in North America, especially in northern Canada, and decreases only in the middle latitudes of Eurasia. Cloud cover averaged over extratropical North America increases $2 \%$, but decreases $1 \%$ over extratropical Eurasia (see Fig. 2). The logarithm of cloud optical thickness in CM2.0 increases in the warmer climate nearly everywhere in North America and Eurasia. This increase averaged over extratropical North America and Eurasia is about $0.2-0.3$ (see Fig. 2), about one-tenth of the mean springtime value of (2.3). The logarithm of cloud optical thickness in CCSM3 increases in the high latitudes of both Eurasia and North America, but de- 
CM2.0
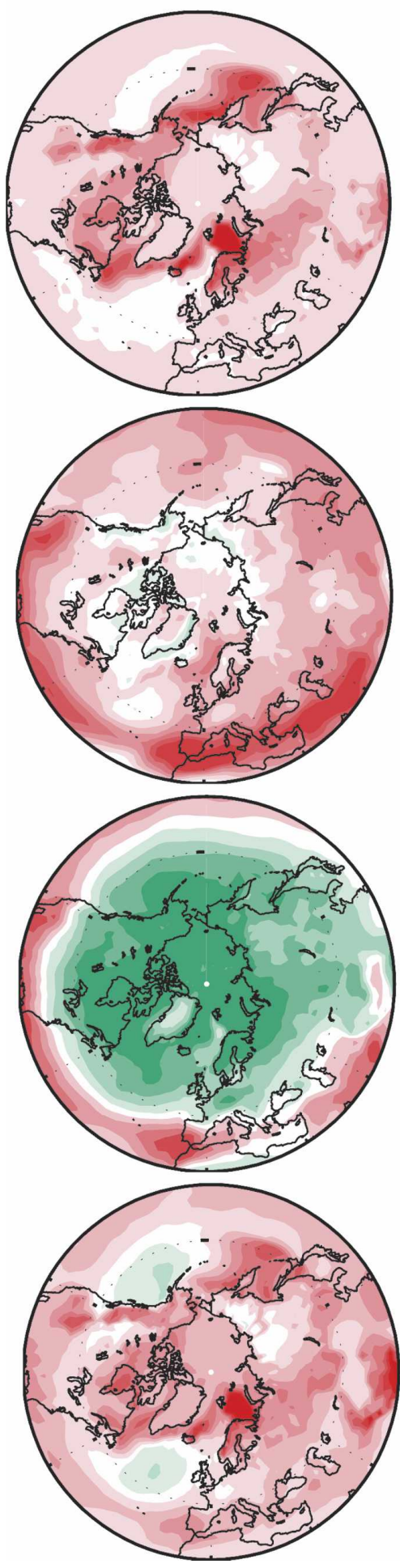

FIG. 1. The geographical distribution of changes in climatological springtime-mean $\alpha_{s}, c$, $\ln (\tau+1)$, and $\alpha_{p}$ from the climate of the twentieth century (1901-2000) to the climate of the twenty-second century (2100-99) in (left) CM2.0 and (right) CCSM3 scenario runs. Here, the changes in $\alpha_{s}, c$, and $\alpha_{p}$ are all evaluated by percentage points (units: \%), rather than a fractional change, and $\ln (\tau+1)$ is in units of tenths.
CCSM3.0
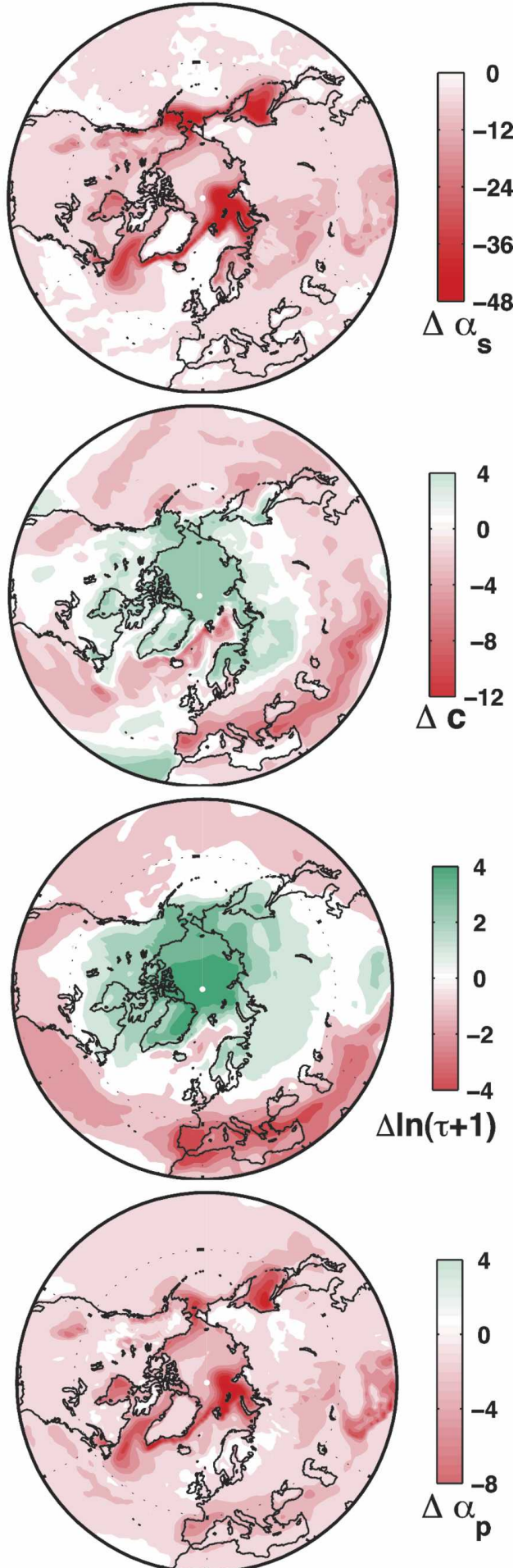

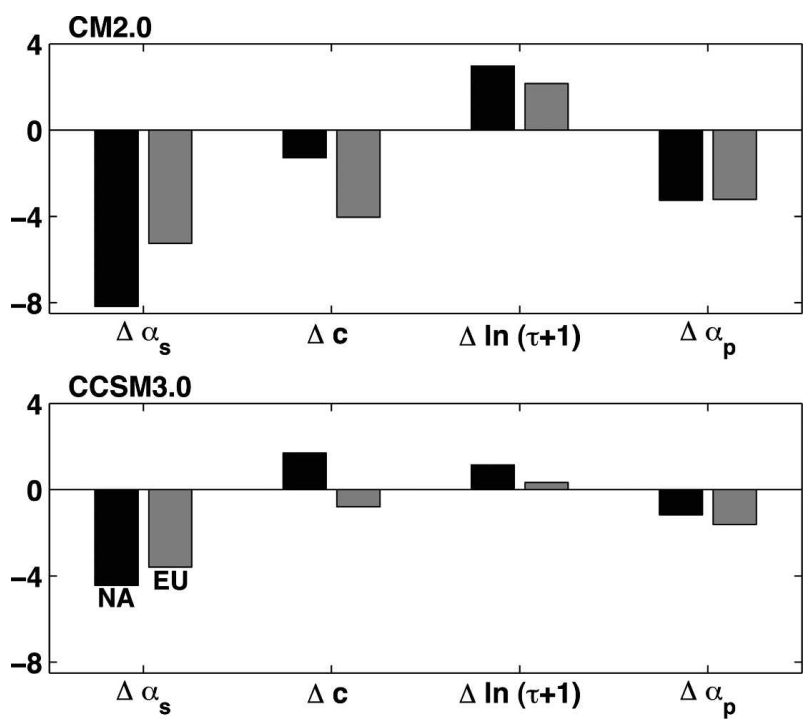

FIG. 2. Changes in climatological springtime-mean $\alpha_{s}, c, \ln (\tau+$ 1 ), and $\alpha_{p}$ averaged over extratropical North America and Eurasia from the climate of the twentieth century (1901-2000) to the climate of the twenty-second century (2100-2199) in (top) CM2.0 and (bottom) CCSM3. In the calculations, changes in the climatological springtime-mean values of $c, \ln (\tau+1)$, and $\alpha_{p}$, as shown in Fig. 1, were weighted by climatological incoming solar radiation at the TOA in the climate of the twentieth century, while changes in the climatological springtime-mean values of $\alpha_{s}$ were weighted by climatological incoming solar radiation at the surface in the climate of the twentieth century. These techniques were used in all calculations involving spatial averages of $c, \ln (\tau+1), \alpha_{p}$, and $\alpha_{s}$ in this article. Note that extratropical North America encompasses the land area northward of $30^{\circ} \mathrm{N}$, including Greenland, and extratropical Eurasia is the Eurasian land area northward of $30^{\circ} \mathrm{N}$. The units for each variable are the same as in Fig. 1.

creases in the middle latitudes. This change averaged over extratropical North America is 0.1, and is slightly positive over extratropical Eurasia (Fig. 2). The disparity in changes in cloud fields between the two models shown in Figs. 1 and 2 is also seen among other AR4 models (not shown). This is consistent with the wide divergence in simulations of cloud feedback among current climate models. We are particularly concerned with this divergence because it complicates our ability

TABLE 2. The changes in the climatological springtime-mean $\alpha_{s}$ (\%) and $T_{s}\left({ }^{\circ} \mathrm{C}\right)$ averaged over North America and Eurasia from the climate of the twentieth century (1901-2000) to the climate of the twenty-second century (2100-99), and the ratio of the former to the latter for the CM2.0 and CCSM3 datasets.

\begin{tabular}{llccc}
\hline \hline & \multicolumn{1}{c}{ Regions } & $\Delta \alpha_{s}$ & $\Delta T_{s}$ & $\Delta \alpha_{s} / \Delta T_{s}$ \\
\hline CM2.0 & North America & -8.17 & 5.58 & -1.46 \\
& Eurasia & -5.24 & 5.26 & -1.00 \\
CCSM3 & North America & -4.44 & 4.40 & -1.01 \\
& Eurasia & -3.59 & 3.97 & -0.90 \\
\hline
\end{tabular}

to assess snow albedo feedback independently of cloud changes.

Planetary albedo in CM2.0 and CCSM3 decreases everywhere in extratropical North America and Eurasia, especially in regions with strong surface albedo reduction such as northern Canada, western Russia, and the Tibetan Plateau. This demonstrates the effect of snow retreat on the TOA shortwave fluxes. However, the effect of cloud variations is visible in regions with small snow albedo reduction, such as western Europe. As shown in Fig. 2, the planetary albedo reduction averaged over extratropical North America and Eurasia in CM2.0 is approximately $3 \%$, about half the albedo reduction at the surface. The planetary albedo reduction averaged over extratropical Eurasia in CCSM3 is approximately $1.6 \%$, also about half the albedo signature at the surface. The planetary albedo reduction averaged over extratropical North America in CCSM3 is only about $1 \%$, considerably less than that averaged over extratropical Eurasia, despite the fact that the surface albedo reduction over North America is slightly greater than that over Eurasia. It seems likely that the substantial increases in cloud cover and cloud optical thickness over North America are responsible for this disproportionately small reduction in planetary albedo, highlighting the need for a method to quantify the dependence of planetary albedo on surface albedo independently of cloud variations.

\section{An analytical model to understand planetary albedo}

In the previous section, we showed that surface albedo, cloud cover, and cloud optical thickness probably all contribute to the simulated planetary albedo change in the warmer climate in two AR4 transient climate change simulations. To isolate the surface contribution, we develop an analytical model governing planetary albedo, and validate it against simulated and satellitebased datasets. This model relates full-sky planetary albedo to known quantities, including clear-sky planetary albedo, cloud cover, cloud optical thickness, and surface albedo. This allows us to generate an analytical expression for $\partial \alpha_{p} / \partial \alpha_{s}$. We can then compare this quantity among models and satellite-based datasets.

The model is derived as follows: Full-sky planetary albedo can be written as the weighted mean of clear-sky planetary albedo $\alpha_{p}^{\text {cr }}$ and cloudy-sky planetary albedo $\alpha_{p}^{\text {cd. }}$

$$
\alpha_{p}=(1-c) \alpha_{p}^{\mathrm{cr}}+c \alpha_{p}^{\mathrm{cd}} .
$$

In Eq. (4), $\alpha_{p}^{\text {cr }}$ is given as follows:

$$
\alpha_{p}^{\mathrm{cr}}=\alpha_{a}^{\mathrm{cr}}+T_{a}^{\mathrm{cr}} \alpha_{s}
$$


which is the sum of clear-sky atmospheric albedo to incoming solar radiation $\alpha_{a}^{\text {cr }}$, and a surface albedo component, which is the product of $\alpha_{s}$ and an effective clear-sky atmospheric transmissivity $T_{a}^{\text {cr }}$. In Eq. (4), $\alpha_{p}^{\text {cd }}$ is given as follows:

$$
\alpha_{p}^{\mathrm{cd}}=\alpha_{a}^{\mathrm{cd}}+T_{a}^{\mathrm{cd}} \alpha_{s}
$$

which is the sum of cloudy-sky atmospheric albedo to incoming solar radiation $\alpha_{a}^{\text {cd }}$ and a surface albedo component, which is the product of $\alpha_{s}$ and an effective cloudy-sky atmospheric transmissivity $T_{a}^{\mathrm{cd}}$. We model the difference between clear- and cloudy-sky atmospheric albedo as proportional to the logarithm of cloud optical thickness, thereby linking $\alpha_{a}^{\mathrm{cr}}$ in Eq. (5) and $\alpha_{a}^{\mathrm{cd}}$ in Eq. (6):

$$
\alpha_{a}^{\mathrm{cd}}=\alpha_{a}^{\mathrm{cr}}+\varepsilon_{1} \ln (\tau+1) .
$$

In Eq. (7), $\varepsilon_{1}$ is the linear coefficient relating the logarithm of cloud optical thickness to the cloudy-sky atmospheric albedo. Similarly, we model the difference between clear- and cloudy-sky effective transmissivity as proportional to the logarithm of cloud optical thickness, thereby linking $T_{a}^{\mathrm{cr}}$ in Eq. (5) and $T_{a}^{\mathrm{cd}}$ in Eq. (6):

$$
T_{a}^{\mathrm{cd}}=T_{a}^{\mathrm{cr}}-\varepsilon_{2} \ln (\tau+1),
$$

where $\varepsilon_{2}$ is the linear coefficient relating the logarithm of cloud optical thickness to the effective cloudy-sky atmospheric transmissivity.

We plug Eqs. (5)-(8) into Eq. (4), and rearrange it into

$$
\alpha_{p}=\alpha_{p}^{\mathrm{cr}}+c \ln (\tau+1)\left(\varepsilon_{1}-\varepsilon_{2} \alpha_{s}\right) .
$$

We can rewrite Eq. (9) as follows:

$$
\alpha_{p}-\alpha_{p}^{\mathrm{cr}}=\varepsilon_{1} c \ln (\tau+1)-\varepsilon_{2} c \ln (\tau+1) \alpha_{s} .
$$

Because $\alpha_{p}, \alpha_{p}^{\text {cr }}, c, \ln (\tau+1)$, and $\alpha_{s}$ are given in models and observations, values of the two linear coefficients, $\varepsilon_{1}$ and $\varepsilon_{2}$, can be readily obtained by regressing $\alpha_{p}-\alpha_{p}^{\text {cr }}$ onto $c \ln (\tau+1)$ and $c \ln (\tau+1) \alpha_{s}$.

Equation (9) is our analytical model for planetary albedo. To test how well it captures planetary albedo and its temporal and spatial variability, we apply it to ISCCP and the AR4 simulations of the current climate. First, $\alpha_{p}, \alpha_{p}^{\mathrm{cr}}, c, \ln (\tau+1)$, and $\alpha_{s}$ in MAM were averaged to get a yearly time series of springtime-mean values at each location within extratropical North America and Eurasia. The time period used was the twentieth century in the case of the AR4 simulations and the years 1984-2000 for the ISCCP dataset. Then, the difference between full-sky and clear-sky planetary albedo $\left(\alpha_{p}-\alpha_{p}^{\text {cr }}\right)$ was regressed onto $c \ln (\tau+1)$ and $c$ $\ln (\tau+1) \alpha_{s}$ to obtain values of $\varepsilon_{1}$ and $\varepsilon_{2}$. We perform the regression calculation based on concatenated time series of all locations within extratropical North
America and Eurasia, so that we are attempting to capture both interannual and geographical variability in planetary albedo. This provides samples large enough to achieve stable statistics in both satellite datasets and simulations, the size of samples being larger than 8000 . We can then model interannual and geographical variations in planetary albedo by plugging in these values of $\varepsilon_{1}$ and $\varepsilon_{2}$, as well as values of $c, \tau, \alpha_{p}^{\mathrm{cr}}$, and $\alpha_{s}$ into Eq. (9). As an example, Fig. 3 shows the scatterplot of modeled planetary albedo against actual planetary albedo in ISCCP datasets and two simulations: CM2.0 and CCSM3. They almost exactly follow a diagonal line, and the correlation coefficient shown in the right bottom corner of each panel is very close to unity, implying nearly perfect agreement. Equation (9) is also used to model planetary albedo in the climate of the twentysecond century in CM2.0 and CCSM3 by plugging in the values of $\varepsilon_{1}$ and $\varepsilon_{1}$ obtained from the current climate, as well as values of $c, \tau, \alpha_{p}^{\mathrm{cr}}$, and $\alpha_{s}$ in the future climate. Similarly, modeled and simulated planetary albedo in the two models are nearly perfectly correlated (not shown). Equation (9) captures the physical relationship seen in the models and ISCCP dataset among planetary albedo, surface albedo and clouds extremely well, not only in the CCSM3 and CM2.0 simulations, but also in the other AR4 models. Plots for the other AR4 models similar to Fig. 3 (not shown) are almost identical, with a nearly one-to-one correspondence between modeled and simulated planetary albedo.

To reveal physical insights behind Eq. (9), we rearrange it:

$$
\alpha_{p}=\left[\alpha_{a}^{\mathrm{cr}}+\varepsilon_{1} c \ln (\tau+1)\right]+\left[T_{a}^{\mathrm{cr}}-\varepsilon_{2} c \ln (\tau+1)\right] \alpha_{s} .
$$

The first term on the right-hand side of Eq. (11) can be viewed as an effective full-sky atmospheric albedo. It is attributed partly to $\alpha_{a}^{\mathrm{cr}}$ and partly to clouds $\left[\varepsilon_{1} c \ln (\tau+\right.$ 1)]. The second term on the right-hand side of Eq. (11) can be viewed as a surface albedo component of fullsky planetary albedo, which is the product of $\alpha_{s}$ and an effective full-sky atmospheric transmissivity $\left[T_{a}^{\mathrm{cr}}-\varepsilon_{2} c\right.$ $\ln (\tau+1)]$. The latter is attributed partly to $T_{a}^{\mathrm{cr}}$ and partly to clouds $\left[-\varepsilon_{2} c \ln (\tau+1)\right]$. The two terms involving clouds in Eq. (11), $\varepsilon_{1} c \ln (\tau+1)$ and $-\varepsilon_{2} c \ln (\tau+$ 1) $\alpha_{s}$, represent two effects on planetary albedo: 1) clouds increase planetary albedo through their own reflectivity, 2) clouds decrease the surface contribution to planetary albedo by reducing the effective full-sky atmospheric transmissivity. In both cases, the effect is proportional to the product of cloud cover and the logarithm of cloud optical thickness. 


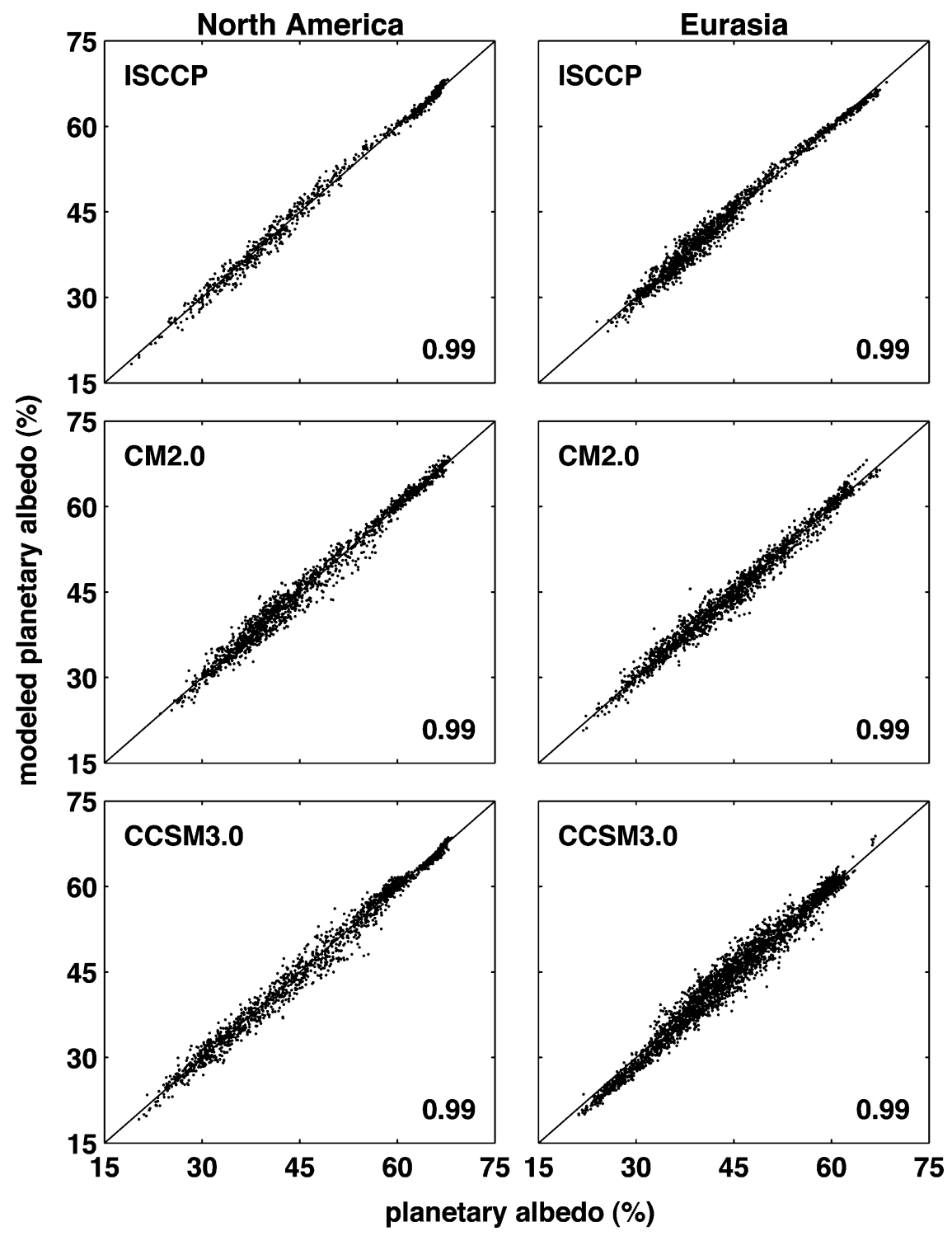

FIG. 3. Scatterplots of modeled interannual and geographical variations in springtime-mean $\alpha_{p}$ based on Eq. (10) and interannual and geographical variations in springtime-mean $\alpha_{p}$ seen in ISCCP, CM2.0, and CCSM3 in extratropical (left) North America and (right) Eurasia for the current climate. The current climate refers to the climate of the 1984-2000 period for ISCCP, and the climate of the twentieth century for CM2.0 and CCSM3. (These same definitions apply to the whole paper.) Here, for clarity, we only show $10 \%$ of ISCCP datasets and $1 \%$ of the two model datasets on this figure. All subsamples are randomly chosen.

\section{Dependence of planetary albedo on surface albedo}

Equation (11) is such a successful model for planetary albedo that we can use it to derive an accurate expression for the first term of Eq. (1), the partial derivative, $\partial \alpha_{p} / \partial \alpha_{\mathrm{s}}$ :

$$
\frac{\partial \alpha_{p}}{\partial \alpha_{s}}=T_{a}^{\mathrm{cr}}-\varepsilon_{2} c \ln (\tau+1) .
$$

The right-hand side of Eq. (12) represents the total attenuation effect of the atmosphere on the surface's contribution to planetary albedo fluctuation. This includes a contribution from the cloudless atmosphere, represented by $T_{a}^{\mathrm{cr}}$, and a contribution from cloud, being proportional to the product of cloud cover and the logarithm of cloud optical thickness. The direct effects of the changes in cloudiness on planetary albedo are not included in Eq. (12), and so are not conflated with the effects of changes in surface albedo, as is the case if the dependence of planetary albedo on surface albedo 


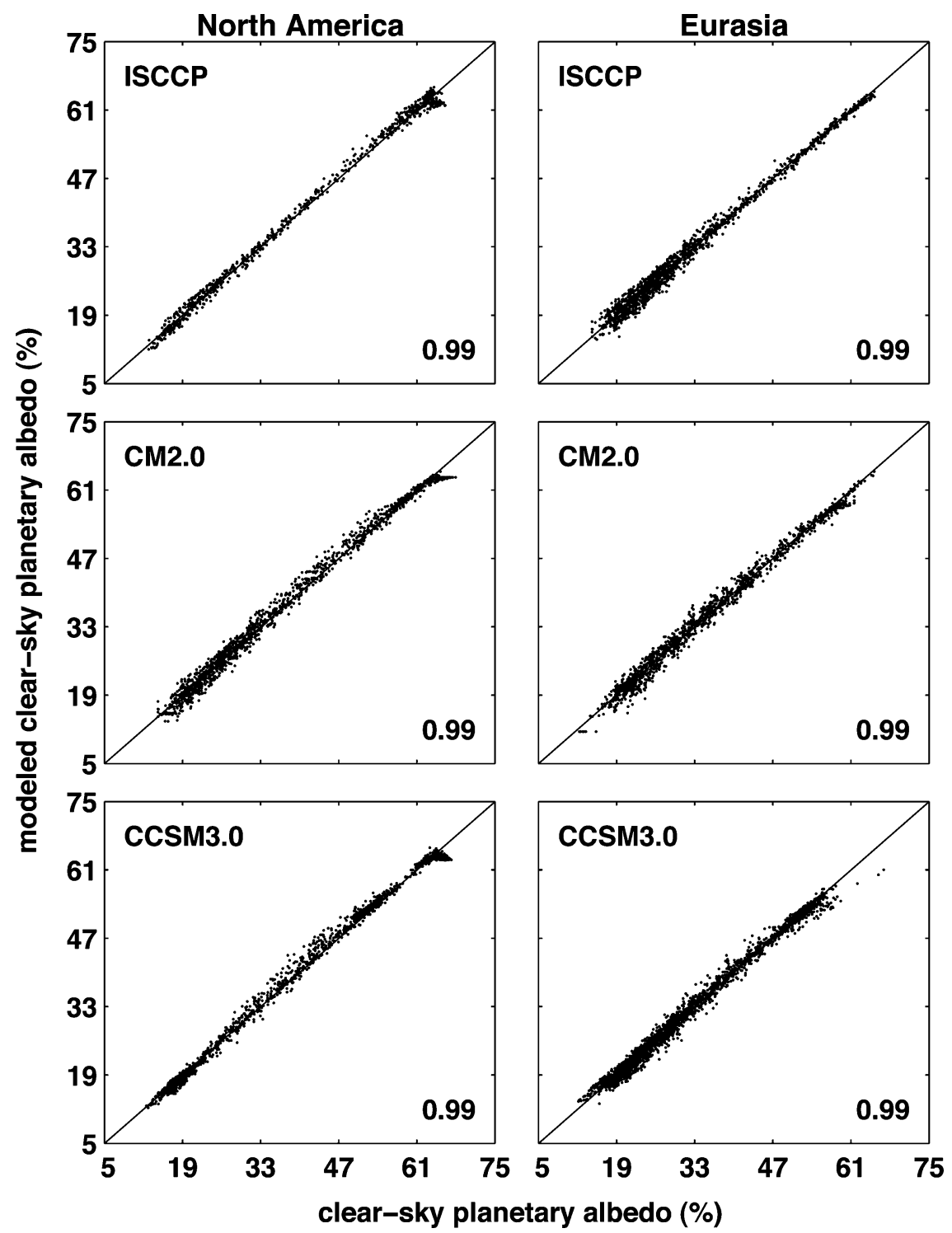

FIG. 4. Scatterplots of modeled interannual and geographical variations in springtime-mean $\alpha_{p}^{\mathrm{cr}}$ and interannual and geographical variations in springtime-mean $\alpha_{p}^{\mathrm{cr}}$ seen in ISCCP, CM2.0, and CCSM3 in extratropical (left) North America and (right) Eurasia for the current climate. The plotting method is the same as in Fig. 3.

is measured by simply regressing planetary albedo variations onto their counterparts at the surface. This points to the relevance of our analytical model in unraveling surface and cloud contributions to planetary albedo variations in cryosphere regions. We will use Eq. (12) to assess the values of $\partial \alpha_{p} / \partial \alpha_{s}$ in the current climate and future climate.

The quantities $c$ and $\tau$ are given by the ISCCP and model datasets, while $\varepsilon_{2}$ is known from regression analysis relying on Eq. (10). The unknown quantity on the right-hand side of Eq. (12) is therefore $T_{a}^{\mathrm{cr}}$. Relying on Eq. (5), we can obtain it by regressing $\alpha_{p}^{c r}$ onto $\alpha_{s}$. In analogy with Fig. 3, we show a scatterplot of modeled clear-sky planetary albedo against the actual clear-sky planetary albedo in ISCCP, CM2.0, and CCSM3 datasets in Fig. 4 as a validation of this regression model. They follow a diagonal line almost exactly and the correlation coefficient, shown in the right bottom corner of each scatterplot, is very close to unity, implying nearly perfect agreement. Figure 5 shows values of $T_{a}^{\mathrm{cr}}$ seen in ISCCP, CM2.0, and CCSM3 in North America and Eurasia. Values of $T_{a}^{\mathrm{cr}}$ in the current climate are comparable among ISCCP, CM2.0, and CCSM3, and in the future climate between CM2.0 and 

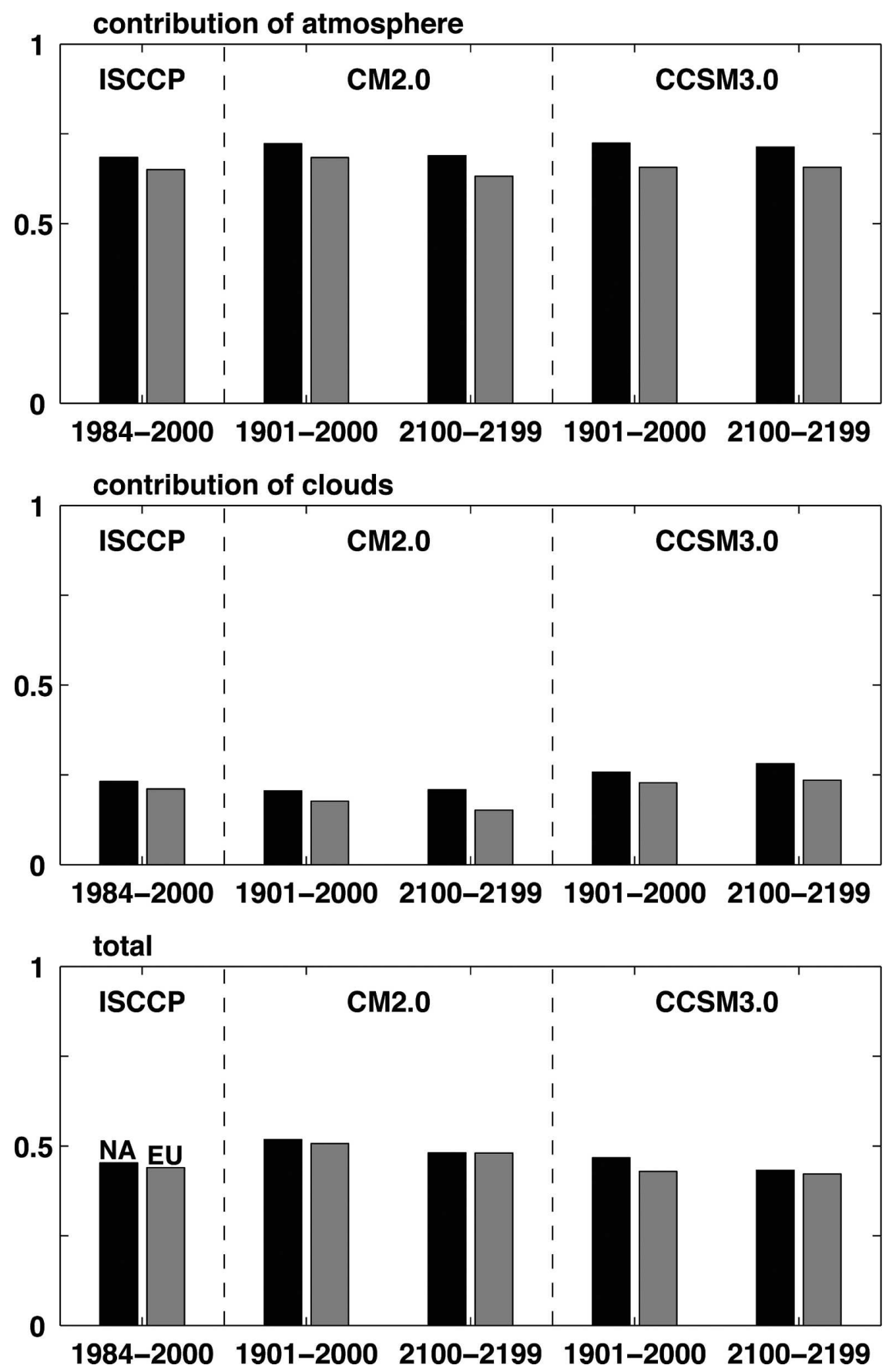

FIG. 5. The climatological springtime-mean values of $T_{a}^{\mathrm{cr}}, \varepsilon_{2} c \ln (\tau+1)$, and $\partial \alpha_{p} / \partial \alpha_{s}$ seen in ISCCP, CM2.0, and CCSM3 in extratropical North America and Eurasia in the current climate and the future climate (2100-99). First, regression calculations are performed to obtain values of $T_{a}^{\mathrm{cr}}, \varepsilon_{1}$, and $\varepsilon_{2}$ based on Eqs. (5) and (10) (see sections 4 and 5). Then, climatological springtime-mean values of $\varepsilon_{2} c \ln (\tau+1)$ are calculated at all locations within extratropical North America and Eurasia. Finally, these values are averaged over extratropical North America and Eurasia, respectively. Note that $\partial \alpha_{p} / \partial \alpha_{s}=T_{a}^{\mathrm{cr}}-\varepsilon_{2} c \ln (\tau+1)$. 
TABLE 3. The climatological springtime-mean values of $\varepsilon_{2}, c$ $\ln (\tau+1)$ in the climate of the twentieth century, and $c \ln (\tau+1)$ in the climate of the twenty-second century for the CM2.0 and CCSM3 datasets over extratropical North America and Eurasia. Also, the climatological springtime-mean values of $\varepsilon_{2}$ and $c \ln (\tau+$ 1) over extratropical North America and Eurasia seen in ISCCP in the current climate (1984-2000).

\begin{tabular}{llccc}
\hline \hline & \multicolumn{1}{c}{ Regions } & $\varepsilon_{2}$ & $\begin{array}{c}c \ln (\tau+1) \\
(1901-2000)\end{array}$ & $\begin{array}{c}c \ln (\tau+1) \\
(2100-99)\end{array}$ \\
\hline CM2.0 & North America & 0.10 & 1.95 & 2.16 \\
& Eurasia & 0.10 & 1.77 & 1.84 \\
CCSM3 & North America & 0.16 & 1.65 & 1.77 \\
& Eurasia & 0.16 & 1.40 & 1.54 \\
ISCCP & North America & 0.14 & 1.51 & - \\
& Eurasia & 0.14 & 1.35 & - \\
\hline
\end{tabular}

CCSM3 in both continents, being about 0.7. This is also true for other simulations (not shown). The clear-sky atmosphere attenuates surface albedo fluctuations so that their magnitudes are consistently reduced by about $30 \%$ as they are mirrored in planetary albedo variations.

According to Fig. 5, there is also a reasonable agreement in values of $\varepsilon_{2} c \ln (\tau+1)$ in the current and future climate in both continents, all being about $1 / 3$ as large as values of $T_{a}^{\mathrm{cr}}$. Values of $\varepsilon_{2} c \ln (\tau+1)$ are relatively small because clouds over the extratropics are generally not very thick [mean value of $\ln (\tau+1)$ is about 2.3] compared with deeper clouds in the Tropics [mean value of $\ln (\tau+1)$ is about 4] and also because a substantial fraction of the sky is clear in $\mathrm{NH}$ snow-covered regions (mean cloud cover is about $60 \%-70 \%$ ). Because values of $\varepsilon_{2}$, the regression coefficient relating cloud optical thickness to cloudy sky transmissivity, are generally smaller than 0.16 (see Table 3), differences in values of $\varepsilon_{2} c \ln (\tau+1)$ in the current and future climate among the three datasets are generally smaller than 0.1 (see Table 3), despite the fact that cloud fields differ somehow in the current and future climate among the three datasets (Table 3).

The mean values of $\partial \alpha_{p} / \partial \alpha_{s}$ seen in ISCCP, CM2.0, and CCSM3 in the current and future climate generally agree, all being about 0.5 (Fig. 5), implying the size of surface-induced planetary albedo fluctuations in the $\mathrm{NH}$ extratropics is about half the albedo signature at the surface. This is the case despite the disparities in cloud fields among ISCCP, CM2.0, and CCSM3, be-
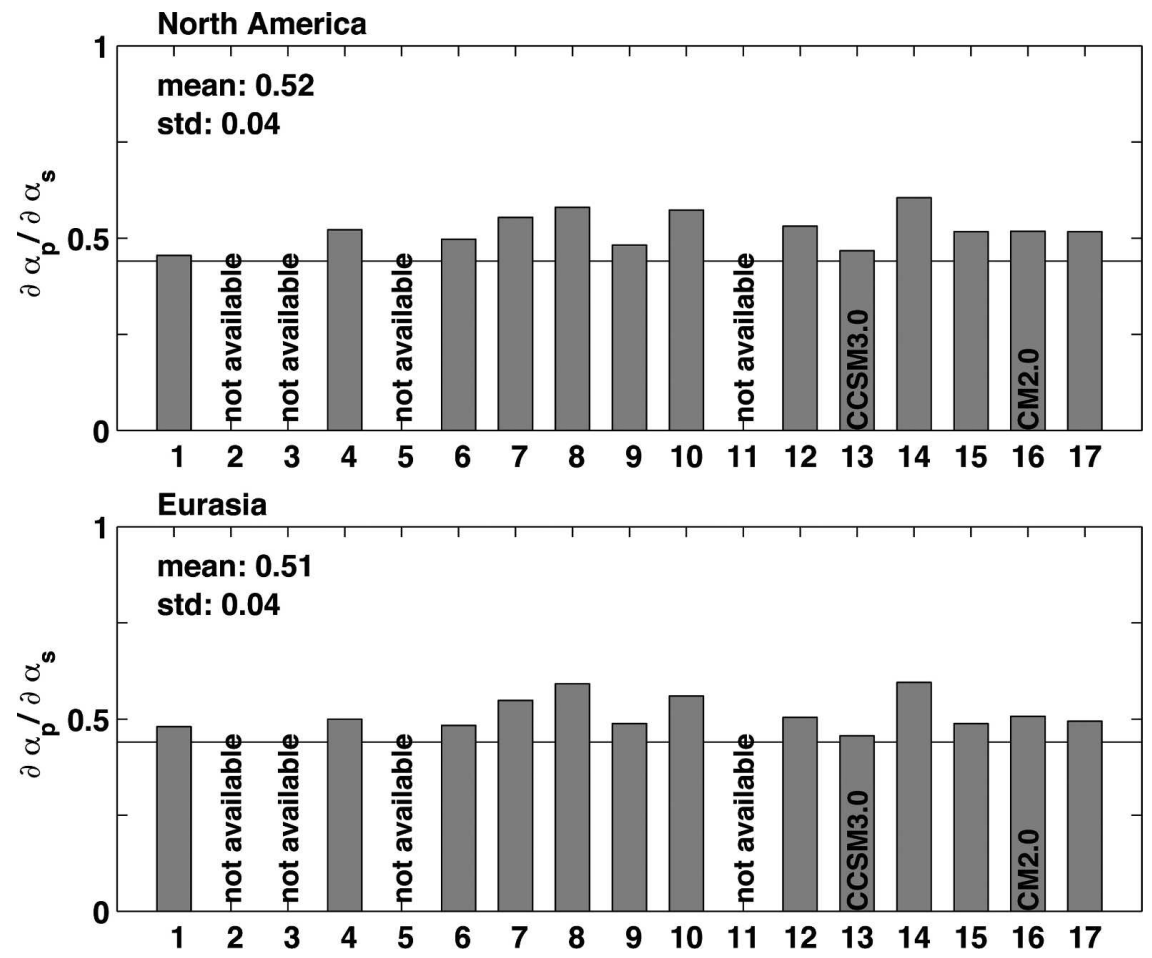

FIG. 6. Values of $\partial \alpha_{p} / \partial \alpha_{s}$ in extratropical (top) North America and (bottom) Eurasia in climate simulations. Procedures similar to those demonstrated in the caption of Fig. 5 are performed to get these values. See Table 1 for the model names. Values of $\partial \alpha_{p} / \partial \alpha_{s}$ are only available for 14 of the 17 models because the other 4 models do not provide all variables required by our analytical model. The solid lines in the figure represent the ISCCP values of $\partial \alpha_{p} / \partial \alpha_{s}$ 

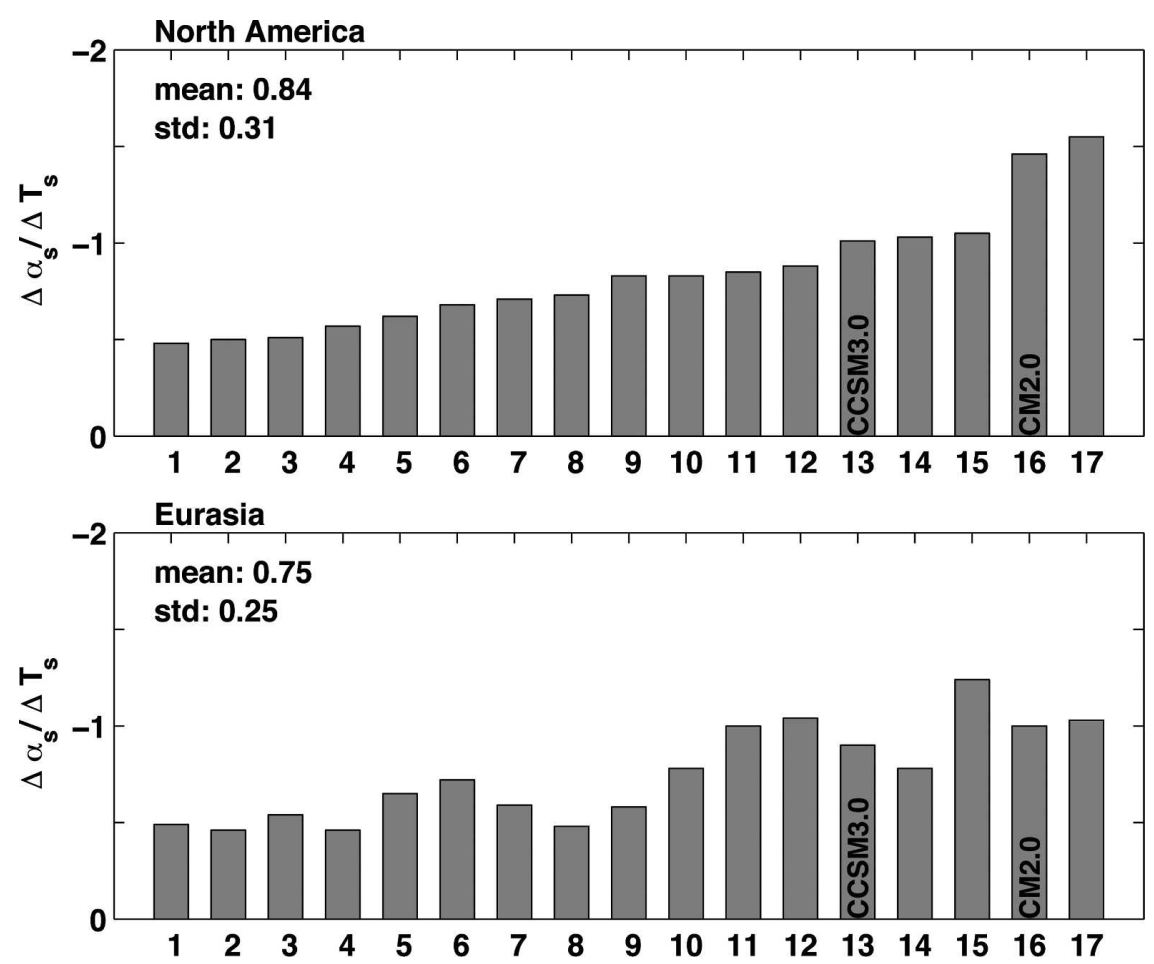

FIG. 7. Values of $\Delta \alpha_{s} / \Delta T_{s}$ (units: $\%{ }^{\circ} \mathrm{C}^{-1}$ ) in extratropical North America and Eurasia in climate simulations. Procedures similar to those demonstrated in the caption of Table 2 are performed to get these values.

tween North America and Eurasia within individual datasets, and within regions going from the present-day to future simulated climates. This demonstrates that disparities in the cloud fields seen in the ISCCP, CM2.0, and CCSM3 are not large enough to generate appreciable divergence in values of $\partial \alpha_{p} / \partial \alpha_{s}$ in the NH extratropics.

Based on Eq. (12), values of $\partial \alpha_{p} / \partial \alpha_{s}$ in the other AR4 simulations are calculated and shown in Fig. 6. The corresponding values in CM2.0 and CCSM3 are also shown in this figure for comparison. Consistent with the agreement between CM2.0 and CCSM3, values of $\partial \alpha_{p} /$ $\partial \alpha_{s}$ are comparable among all the simulations. The standard deviation of this quantity among all the simulations is 0.04 , only about $8 \%$ of the mean value (0.52) in both North America and Eurasia. Moreover, in the majority of the simulations, values of $\partial \alpha_{p} / \partial \alpha_{s}$ agree with the ISCCP values (represented by the solid lines in Fig. 6) to within $10 \%$. This indicates that the $\partial \alpha_{p} / \partial \alpha_{s}$ component of snow albedo feedback simulated in climate models agrees quite well with observations.

\section{Summary and implications}

The strength of snow albedo feedback can be quantified as the product of two terms, one representing the dependence of planetary albedo on surface albedo $\left(\partial \alpha_{p} /\right.$ $\left.\partial \alpha_{\mathrm{s}}\right)$ and another representing the change in surface albedo induced by a unit temperature change $\left(\Delta \alpha_{s}\right)$ $\left.\Delta T_{s}\right)$. The $\partial \alpha_{p} / \partial \alpha_{s}$ term is the variation in planetary albedo with surface albedo in NH extratropical land areas, with other factors such as water vapor concentration and cloud fixed. However, cloud properties can change substantially over the course of transient climate change experiments, and these can in turn have a large effect on incoming solar radiation. This makes it particularly difficult to quantify the strength of this aspect of snow albedo feedback.

To isolate the surface contribution from that of cloud, we develop an analytical model governing planetary albedo. This model relates full-sky planetary albedo to known quantities, including clear-sky planetary albedo, cloud cover, cloud optical thickness, and surface albedo. It captures both interannual and geographical variability in planetary albedo in simulated and satellite-based datasets extremely well. The advantage of this model is that we can use it to derive an expression for a true partial derivative of planetary albedo with respect to surface albedo. It includes a contribution from the cloudless atmosphere, represented by an effective clear-sky atmospheric transmissivity as 
well as a contribution from cloud, being proportional to the product of cloud cover and logarithm of cloud optical thickness.

There is good agreement in values of the clear-sky component of the atmosphere's attenuation of surface albedo anomalies, both in the current climate and in the future climate in both North America and Eurasia, all being about 0.7 . Values of the cloud contribution to the variation in planetary albedo with surface albedo are about $1 / 3$ the size of the clear-sky component. Cloud is not the most significant factor controlling the variation in planetary albedo with surface albedo both because clouds over the extratropics are generally not very thick and because a substantial fraction of the sky is clear in $\mathrm{NH}$ snow-covered regions. Therefore, differences or errors in mean cloud fields of the magnitude seen in the current generation of climate models are not large enough to cause appreciable divergence or errors in estimates of the variation of planetary albedo with surface albedo in the current climate. For example, differences in values of the cloud contribution to the atmospheric attenuation effect among the three datasets in the current climate are generally only about $10 \%$ of the total attenuation effect. Nor are changes in mean cloud fields as a result of climate change large enough to cause the variation of planetary albedo with surface albedo to exhibit significant time dependence. Because of this relative insensitivity to variations in mean cloud fields, the mean values of the variation of planetary albedo with surface albedo seen in the simulated current and future climate are in general agreement, all being about 0.5 . This value is also in agreement with the satellite-based ISCCP dataset. Therefore we can say with a high degree of confidence that both in simulations and satellite-based datasets, snow-induced planetary albedo anomalies are about half the albedo signature at the surface.

Finally, armed with these conclusions about the $\partial \alpha_{p} /$ $\partial \alpha_{s}$ term in Eq. (1), we focus on the second term in this equation: $\Delta \alpha_{s} / \Delta T_{s}$. This term is related to surface processes and represents the sensitivity of surface albedo to surface air temperature in the NH extratropics. It is easily quantified in AR4 climate simulations, and we show the results in Fig. 7. This quantity varies a great deal from model to model. The standard deviation of this quantity among all the simulations is about $1 / 3$ of the mean value in both North America and Eurasia, much greater than the relative variation in $\partial \alpha_{p} / \partial \alpha_{s}$ shown in Fig. 6. And over both North American and Eurasia, the largest value is approximately 3 times as large as the smallest. Therefore, we determine that this term is unquestionably the main source of the large divergence in simulations of snow albedo feedback. To reduce this divergence, attention should be focused on differing parameterizations of surface processes rather than intermodel variations in the attenuation effect of the atmosphere on surface albedo anomalies.

Acknowledgments. This study is based on model integrations performed by NCAR and CRIEPI with support and facilities provided by NSF, DOE, ESC/ JAMSTEC, and MEXT. The authors acknowledge the international modeling groups for providing their data for analysis, the Program for Climate Model Diagnosis and Intercomparison for collecting and archiving the model data, the JSC/CLIVAR Working Group on Coupled Modelling and their Coupled Model Intercomparison Project and Climate Simulation Panel for organizing the model data analysis activity, and the IPCC WG1 TSU for technical support. The IPCC Data Archive at Lawrence Livermore National Laboratory is supported by the Office of Science, U.S. Department of Energy. This study was supported by NSF Grant ATM0135136, but any opinions, findings, and conclusions or recommendations expressed in this material are those of the authors and do not necessarily reflect the views of the National Science Foundation. The authors thank Y.-C. Zhang for his help with the ISCCP cloud and flux datasets and two anonymous reviewers for their constructive criticism of this manuscript.

\section{REFERENCES}

Briegleb, B. P., C. M. Bitz, E. C. Hunke, W. H. Lipscomb, M. M. Holland, J. L. Schramm, and R. E. Moritz, 2004: Scientific description of the sea ice component in the Community Climate System Model, version three. Tech. Note NCAR/TN$463+$ STR, 70 pp.

Cess, R. D., and G. L. Potter, 1988: A methodology for understanding and intercomparing atmospheric climate feedback processes in general circulation models. J. Geophys. Res., 93 (D7), 8305-8314.

- , and Coauthors, 1991: Interpretation of snow-climate feedback as produced by 17 general circulation models. Science, 253, 888-892.

Collins, W. D., and Coauthors, 2004: Description of the NCAR Community Atmosphere Model (CAM3.0). Tech. Note NCAR/TN-464+STR, 214 pp.

Cubasch, U., and Coauthors, 2001: Projections of future climate change. Climate Change 2001: The Scientific Basis, J. W. Kim and J. Stone, Eds., Cambridge University Press, 525-582.

GFDL Global Atmosphere Model Development Team (GAMDT), 2004: The new GFDL global atmosphere and land model AM2/CM2.0: Evaluation with prescribed SST simulations. J. Climate, 17, 4641-4673.

Groisman, P. Y., T. R. Karl, and R. W. Knight, 1994a: Observed impact of snow cover on the heat balance and the rise of continental spring temperatures. Science, 263, 198-200.

,-- , and $-1994 \mathrm{~b}$ : Changes of snow cover, temperatures and radiative heat balance over the Northern Hemisphere. $J$. Climate, 7, 1633-1656. 
Hall, A., 2004: The role of surface albedo feedback in climate. $J$. Climate, 17, 1550-1568.

Holland, M. M., and C. M. Bitz, 2003: Polar amplification of climate change in coupled models. Climate Dyn., 21, 221-232.

Milly, P. C. D., and A. B. Shmakin, 2002: Global modeling of land water and energy balances. Part I: The land dynamics (LaD) model. J. Hydrometeor., 3, 283-299.

Oleson, K. W., and Coauthors, 2004: Technical description of the Community Land Model (CLM). Tech. Note NCAR/TN464+STR, 174 pp.

Prentice, I. C., and Coauthors, 2001: The carbon cycle and atmospheric carbon dioxide. Climate Change 2001: The Scientific Basis, L. Pitelka and A. Ramirez Rojas, Eds., Cambridge University Press, 183-237.

Randall, D. A., and Coauthors, 1994: Analysis of snow feedbacks in 14 general circulation models. J. Geophys. Res., 99, 20 75720772.

Rossow, W. B., and R. A. Schiffer, 1991: ISCCP cloud data products. Bull. Amer. Meteor. Soc., 72, 2-20.

, and L. C. Garder, 1993a: Cloud detection using satellite measurements of infrared and visible radiances for ISCCP. $J$. Climate, 6, 2341-2369.

- and,- 1993 b: Validation of ISCCP cloud detections. $J$ Climate, 6, 2370-2393.

- and R. A. Schiffer, 1999: Advances in understanding clouds from ISCCP. Bull. Amer. Meteor. Soc., 80, 2261-2287.

- A. W. Walker, and L. C. Garder, 1993: Comparison of ISCCP and other cloud amounts. J. Climate, 6, 2394-2418.

,-- D. Beuschel, and M. Roiter, 1996: International Satellite Cloud Climatological Project (ISCCP) description of new cloud datasets. Tech. Doc. WMO/TD 737, World Climate Research Programme (ICSU AND WMO), 113 pp.

Smith, R., and P. Gent, 2004: Reference Manual for the Parallel Ocean Program POP: Ocean System Model (CCSM2.0 and 3.0). National Center for Atmospheric Research, 75 pp.

Zhang, Y.-C., W. B. Rossow, A. A. Lacis, V. Oinas, and M. M. Mishchenko, 2004: Calculation of radiative fluxes from the surface to top of atmosphere based on ISCCP and other global datasets: Refinements of the radiative transfer model and the input data. J. Geophys. Res., 109, D19105, doi:10.1029/ 2003JD004457. 